\title{
Lifestyle exercises for bone health and health-related quality of life among premenopausal women: a randomised controlled trial
}

\author{
Opeyemi Babatunde ${ }^{1}$ and Jacky Forsyth ${ }^{2}$
}

\begin{abstract}
:
Purpose: Growing evidence supports engagement in physical exercise throughout life for optimal bone health. However, promotion of physical exercise among premenopausal women presents significant challenges, which are yet to be addressed in the design of many bone-health exercise regimes. As a deviation from traditional, facility-based, supervised, long-duration and intense exercise regimes, the efficacy of short-duration, practical, lifestyle physical exercises for improving bone health and quality of life was examined.

Methods: Premenopausal women $(N=96$, mean age $22.25 \pm 3.5$ years; mean BMI $23.43 \pm 3.5 \mathrm{~kg} /$ $\mathrm{m}^{2}$ ) participated in a 6-month randomised trial of lifestyle physical exercises for the intervention group and sham exercises for the control group. The participants' scores on the outcome measure SF-36 was accessed pre- and post-intervention and compared with general population norms according to the SF-36 scoring manual. Paired $t$-tests were used to examine changes within trial arms from baseline to post-intervention, while analysis of covariance was performed to examine the effect of the lifestyle exercise programme on quality of life of premenopausal women.

Results: Compared to $51 \%$ at baseline, $63 \%$ of the participants were either at or above the general population norm for general health, and the percentage of participants who were below the population health norm for mental health was reduced from $46 \%$ at baseline to $38 \%$ post-exercise intervention. Comparable improvements in quality of life were found in both trial arms post-participation in the bone-health promotion programme.

Conclusions: Bone-health exercises, when implemented as easily adoptable, lifestyle physical activity, may also enhance the quality of life of premenopausal women. Hence, a practical lifestyle approach to exercise may offer a much-needed public health strategy for bone-health promotion among women.
\end{abstract}

Keywords: bone-health promotion, physical activity, lifestyle exercise, quality of life, women

\section{Introduction}

As a patient-oriented outcome measure, healthrelated quality of life (QOL) is increasingly pivotal to trials investigating the effectiveness of interventions (1). Considering its physiological and psychosocial effects, epidemiological evidence and biological plausibility designate a positive relationship between physical exercise and QOL in clinical and nonclinical populations (2). Similarly, bone-loading (i.e. osteogenic) exercises may not be restricted to bringing about systemic changes to bone alone, as such modes of physical exercise have been positively associated with QOL across groups of people with osteoporosis $(3,4)$.

1. Research Institute for Primary Care \& Health Sciences, Keele University, UK.

2. School of Psychology, Sport and Exercise, Faculty of Health Science, Staffordshire University, Stoke-on-Trent, UK.

Correspondence to: Opeyemi Babatunde, Arthritis Research UK Primary Care Centre Research Institute for Primary

Care \& Health Sciences, Keele University, Staffordshire ST5 5BG, UK. Email: o.babatunde@keele.ac.uk

(This manuscript was submitted on 23 May 2014. Following blind peer review, it was accepted for publication on 6 November 2014)

Global Health Promotion 1757-9759; Vol 0(0): 1-9; 568901 Copyright (C) The Author(s) 2015, Reprints and permissions: http://www.sagepub.co.uk/journalsPermissions.nav DOI: 10.1177/1757975914568901 http://ghp.sagepub.com 
Osteoporosis, formerly considered as a natural consequence of ageing, is now known to be largely preventable, but the global burden of osteoporosis, and the considerable morbidity, mortality and escalating health care costs associated with it, is still on the rise $(5,6)$. In spite of the significant advances that have been made in the field of bone health and exercise research, pragmatic reduction of its incidence and the burden of prevalence are still subject to evolving research. The reason for the high incidence and prevalence of osteoporosis may be due partly to the current definitions and execution of primary preventative strategies (7). One easily noticeable result of such systemic failure is the low level of advocacy and actual engagement in physical exercise across the lifespan $(8,9)$.

Furthermore, the current widespread use of a strict laboratory-based approach to examining the effects of exercise on bone health might negatively impact the process of designing truly effective exercise programmes for enhancing bone health. Findings from such laboratory- or exercise facilitybased investigations may be difficult to translate into real-world settings where continuous or lifetime commitments to engagement in osteogenic exercises do not exist in a vacuum. In reality, commitments to engagement in exercises are influenced by many factors (such as work commitments, financial obligations and access to exercise facilities), which may not always favour long-term participation in rigorous regimes like those employed in many trials.

One other important issue affecting the translation of exercise and bone-health research into clinical osteogenic benefit may be the high level of exertion that is required to engage in currently recommended bone-health exercises, which are also longer in duration per session (5). Particularly among the premenopausal age group, promotion of bonehealth-enhancing physical exercise presents with significant barriers (10-12). For instance, women's perception of physical exercises as rigorous, timeconsuming and of moderate efficacy has been shown to negatively impact their intention to engage in and participate in exercise-related interventions for bone health $(9,10,12-15)$.

Since strong evidence suggests that women may lose bone mineral density prior to menopause (1619), public health considerations for improving bone health at this phase of life must be targeted if the prevalence and impact of skeletal system dysfunctions in women is to be reduced. The key to the challenge of getting women to exercise may subsequently lie in careful consideration of the psychosocial correlates of engagement in physical activity (such as age, access to exercise facilities, time constraints, and motivation and/or perceived barriers to physical exercise) while designing exercise-related interventions for bone health (14).

To improve compliance and adherence, it is logical that exercise prescriptions for bone health should be easily adoptable, have osteogenic efficacy, and be suitable and sustainable for the population for which they are being specified. Bone health promotion behaviours of increased physical exercise uptake and adherence may be improved when the population perceives that bone health exercise prescriptions confer added value in terms of overall satisfaction with life (12).

In a bid to investigate the effectiveness of a regime of non-facility-based osteogenic exercises for the primary prevention of osteoporosis among premenopausal women, this study examined the relative effect of bone health exercises on quality of life. It was hypothesised that alongside bone health augmentation, the QOL of premenopausal women could be enhanced with short, discrete bouts of osteogenic exercises which are easily adoptable into the lifestyle. Hence, we report on the potential of lifestyle physical exercises which were aimed at promoting bone health to also bring about changes in health-related QOL of premenopausal women.

\section{Methods}

The protocol regarding the recruitment, instruments and physical exercise intervention for this study follows earlier published and validated designs (20-23). Briefly, ethical approval for the study was obtained from the local research ethics committee (09/H1204/76) and the institutional ethics review board, and all participants gave written informed consent prior to participation. Volunteers were screened to exclude women who were younger than 18 years or older than 35 years, significantly obese as determined by baseline body mass index $(\mathrm{BMI})>35 \mathrm{~kg} / \mathrm{m}^{2}$, those with current or recent history (previous 12 months) of medical or surgical problems likely to affect bone metabolism, and a family history of osteoporosis. Women were also excluded for pregnancy, recent childbirth, current or 


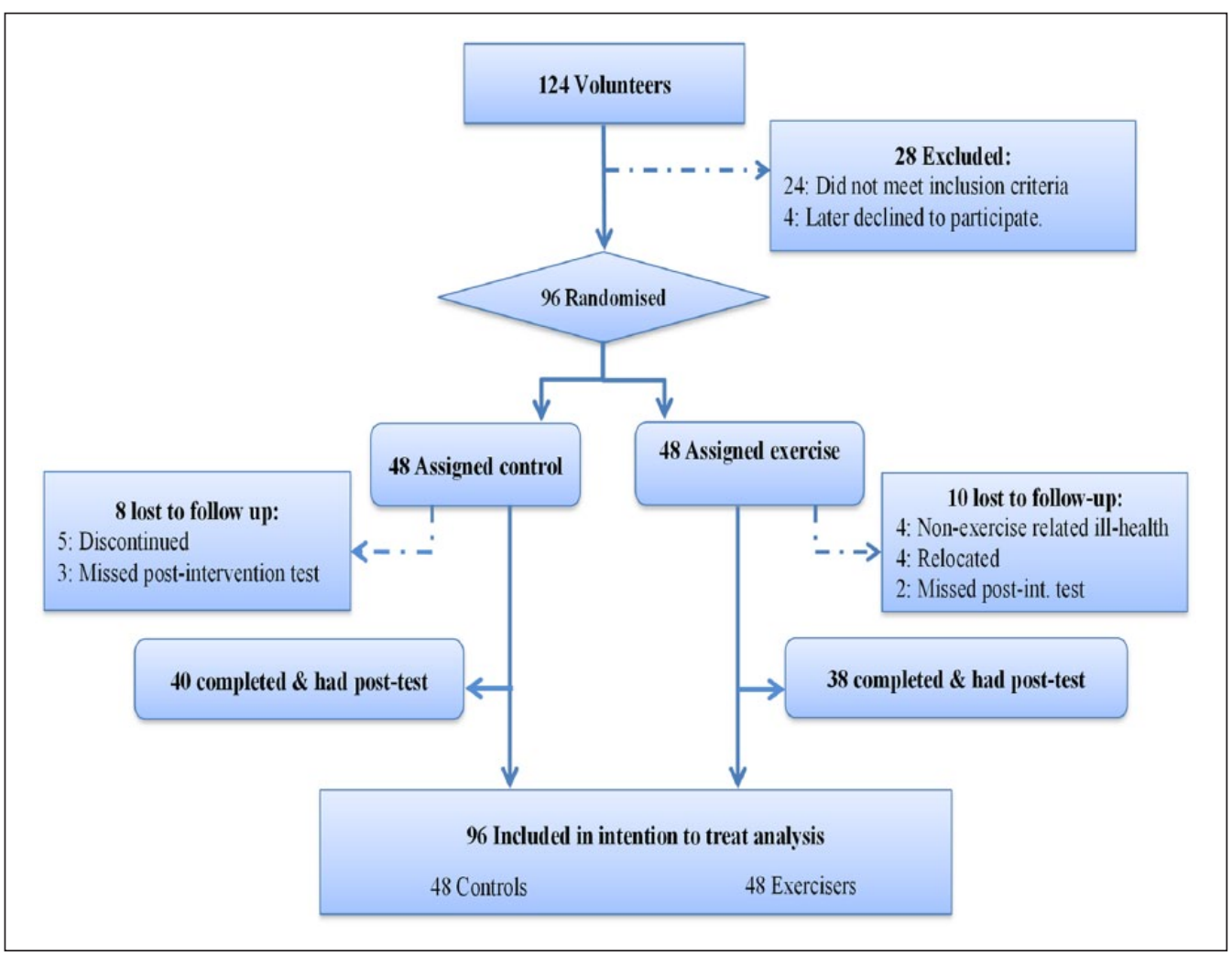

Figure 1. Flow chart of the study.

recent (previous 6 months) lactation and hysterectomy. A total of 96 premenopausal women (mean age $22.25 \pm 3.5$ (range 18-35) years; mean BMI $23.43 \pm 3.5($ range $\left.17.7-32.8) \mathrm{kg} / \mathrm{m}^{2}\right)$ who met the inclusion criteria were blindly randomised into exercise (48) and sham exercise (48) groups by random sequence generation. A flow chart of the study is presented in Figure 1.

As a means of increasing compliance and adherence to the physical exercise regime during the trial and beyond the study's lifespan, specific psychosocial concepts for engagement in lifestyle physical exercises were taken into consideration. Such concepts include personal regulation of goaldirected health behaviour (self-control), selfconfidence (despite absence of facility-based supervision) and reinforcement. Also, the programme drew on Bandura's Social Cognitive Theory (SCT) for changing behaviour (21). In relation to the identified constructs, strategies for uncompromising commitment to engage in the exercise irrespective of probable circumstances such as bad weather, time constraints, stress, and fatigue were discussed with each participant. Motivating factors and perceived adherence to lifestyle physical exercise for bone health were examined in relation to each participant's own personal circumstances. The participants were then given the opportunity to design personalised adherence strategies and goal-setting. According to the approach advocated by Dishman et al. (22), precautions were taken to foster self-efficacy in both exercise and sham exercise groups.

\section{Exercise intervention}

Based on the evidence for the osteogenic effectiveness of short, discrete bouts of restinterspersed, high-impact exercise for mature bone (23), this trial was designed to load the bone of premenopausal women with a few loading cycles of 
mechanical strain for optimum osteogenic response. In the exercise intervention group, participants performed two-legged maximum vertical jumps 10 times using an arm swing in countermovement jump style, barefooted on a relatively hard floor, three days per week for a period of 6 months. To ensure quality control, digitised video clips of the physical exercises were given to all participants (intervention group), who were encouraged to perform the physical exercises as demonstrated in the video. Similarly, leaflets describing bespoke (stretching) exercises with detailed instructions and diagrams were given to the sham exercise group participants. Stretching has not been reported to promote osteogenic activity (24); hence, the sham exercise group participants performed bouts of sham exercises three days per week for 6 months. Since participants were blinded to randomisation, engaging the sham exercise group in a regular physical exercise programme was designed to foster the perception of full participation in the study, as well as decreasing attrition in this trial arm. Participants were requested to maintain their usual diet and lifestyle throughout the study. All participants were free to choose where and when to do the exercises and were provided with individual training $\operatorname{logs}$ to record the amount of exercise completed as honestly as possible. Compliance and adherence to the exercise regime during the intervention were monitored via telephone calls, text messages and emails (at four-week intervals) to all participants. Participants were also free to contact the researcher in the event of any issues pre-, during and post-exercise intervention.

\section{Health-related QOL}

Health-related QOL was assessed at baseline and at 6 months post-intervention with the aid of the SF-36v2 Health Survey, a generic QOL instrument (25), which has also been employed to assess the physical and mental components of health-related QOL following physical exercise interventions. The SF-36v2 Health Survey contains 36 items within eight domains that measure functional health and well-being from the respondent's perspective. Participants' scores on each scale were calculated according to the SF-36 scoring manual compared with population norms for each health domain (26). The eight domains (i.e. physical functioning, role-physical, role-emotional, social functioning, bodily pain, mental health, vitality and general health perceptions) were categorised into QOL component scales: the physical component summary (PCS) and mental component summary (MCS). A third global score, SF-6D or Utility index (UT Index), scored from 0.0 (worst health state) to 1.0 (best health state), was also created.

\section{Data analysis}

Descriptive and exploratory data analyses were performed. Baseline characteristics were compared between exercise and sham exercise groups by independent $t$-tests. The intervention effect of physical exercise was estimated with an intentionto-treat (ITT) approach, whereby missing data were estimated with a last-observation-carried-forward approach (using the baseline data of those who dropped out). Paired-samples $t$-tests were conducted to examine the change in mean QOL scores of participants' pre- and post- intervention within each trial arm. Analyses of covariance (ANCOVA) determined whether the 6-month regime of brief rest-inserted bouts of osteogenic exercises would significantly enhance the QOL of premenopausal women. In the ANCOVA analyses, participants' change in QOL scores (PCS, MCS, UT Index) from pre- to post-intervention QOL scores were the outcome variables, the intervention group (exercise vs sham exercise) served as independent variables, while participants' baseline scores were entered into the model as covariates. All of the assumptions for ANCOVA were met. Confidence interval and the $p$ value were set at $95 \%$ and $<.05$, respectively. Data were analysed using Windows SPSS version 21.0.

\section{Results}

Study participants were all of Caucasian ethnic origin. There was no significant difference among exercise intervention and sham exercise groups post-randomisation for measured parameters (i.e. age, BMI, QOL, bone health and dietary calcium intake) at baseline (Table 1). All the participants ranged from being sedentary (i.e. engaged in no form of regular exercise in a typical week) to being moderately active (i.e. reported exercising regularly on not more than three days per week) for at least 6 months prior to the commencement of the study. 
Table 1. Demographic/baseline characteristics of participants.

\begin{tabular}{lccc}
\hline & Exercise $(n=48)$ & Sham exercise $(n=48)$ & $p$-value \\
\hline Age $($ years $)($ mean $\pm \mathrm{SD})$ & $22.8 \pm 4.0$ & $21.7 \pm 2.9$ & 0.11 \\
BMI $\left(\mathrm{kg} / \mathrm{m}^{2}\right)($ mean $\pm \mathrm{SD})$ & $23.5 \pm 3.5$ & $23.4 \pm 3.6$ & 0.94 \\
BUA $(\mathrm{dB} / \mathrm{MHz})($ mean $\pm \mathrm{SD})$ & $64.3 \pm 4.9$ & $64.4 \pm 4.5$ & 0.89 \\
FFQ score $($ mean $\pm \mathrm{SD})$ & $1726 \pm 1045$ & $1392 \pm 798$ & 0.09 \\
\hline
\end{tabular}

BMI: body mass index; BUA: Broadband ultrasound attenuation (assessing bone health); FFQ score: food frequency questionnaire score (estimates calcium intake).

Although there was a report of short-term illness (non-study-related) by three participants during the study, participants reported being in a general state of good health before and after participating in the study, and there was no incident of adverse events as a direct result of participation.

At the post-intervention evaluation, up to $68 \%$ of the participants registered their intention to continue with the physical exercise regime and used phrases such as:

'I do it with my flat mate and it helps!' (a sham exercise group participant)

'I think I am going to continue because I enjoyed it, doing the exercises sometimes helps me to feel relieved of stress' (an exercise intervention group participant)

For many of the participants, the freedom to adapt the exercise programme to personal schedules was a perceived advantage and motivating factor for continued engagement. The study retention was $81 \%(n=78)$, as 18 missing cases of post-intervention data were lost to dropout and failure to turn up for post-intervention assessments. At the end of the trial, reported exercise compliance was $80 \%(\sim 2.5$ sessions per week) for both exercise and sham exercise groups $(t(94)=-0.155, p=0.878)$.

After 6 months of the lifestyle physical exercise programme, marginal improvement in physical and mental health states was achieved by study participants (both exercise and sham exercise groups). The mean changes in physical and mental health-related QOL scores relative to baseline values are presented in Table 2 . Of the 78 women who were assessed post-intervention, $63 \%$ of them were either at or above the general population norm for general health, compared to $51 \%$ of them when they were assessed at baseline prior to participation in the study. Similarly, the percentage of participants who were below the population health norm for mental health reduced from $46 \%$ at baseline to $38 \%$ postexercise intervention. Across all the eight domains of health-related QOL, exercise and sham exercise groups were similar $(p>0.05)$. Furthermore, in the analyses of exercise intervention effects by ANCOVA, there was no statistically significant effect of engagement in the exercise intervention on the $\operatorname{MCS}\left(F(1,93)=0.790, p=0.376, \eta^{2}=0.008\right)$, $\operatorname{PCS}\left(F(1,93)=0.33, p=0.565, \eta^{2}=0.004\right)$, and UT Index $\left(F(1,93)=0.539, p=0.465, \eta^{2}=0.006\right)$ of the premenopausal women. The pre-intervention QOL measures (MCS, PCS and UT Index) were not significantly related to the change scores postintervention. Hence, after controlling for baseline MCS, PCS and UT Index scores, the improvements in QOL due to participation in lifestyle physical exercise were comparable for both exercise and sham exercise groups.

\section{Discussion}

\section{Lifestyle physical exercise and health-related QOL}

The results of this study show that short discrete bouts of lifestyle physical exercise are easily adoptable and may enhance QOL of premenopausal women. The apparent increase in health-related QOL of the premenopausal women who participated in this study corroborates long-established perceived health benefits of physical exercise (27).

The study participants were blinded to the assignment of exercise intervention, and the sham exercises were designed to mimic the osteogenic exercises that were prescribed for the exercise intervention group in terms of duration, in-built short discrete bouts and frequency of engagement, such that the physical exercise for the intervention and placebo control arms of the trial varied only in 


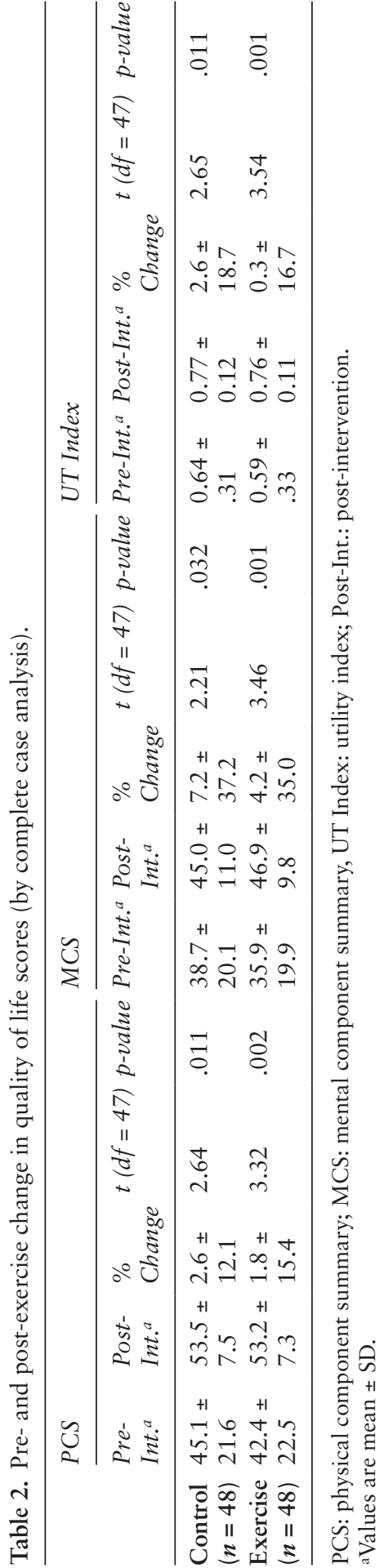

type. However, the 6-month regime of lifestyle physical exercise led to some (though small) improvement in mental and physical health-related QOL of participants in both exercise intervention and sham exercise groups. The improvement in QOL of the sham exercise group, who had been given bespoke sham exercises to do during the 6-month period, may be attributed to a placebo effect, since such types of sham exercises have not been known to promote osteogenic activity (24).

The perceived boost in health status may have been one of the enduring factors for compliance and adherence to the bone health programme in both groups. This finding bears important implications regarding the feasibility of preventative and/or targeted health promotion programmes in the future, because people are more likely to comply with programmes for which they perceive additional health benefits. Conversely, the lack of appreciable increase in QOL status of participants in this study may have been accounted for by the absence of group effects and contact with physical activity and health experts on a frequent basis during the regime, as the programme was non-facility-based and nonsupervised. Group and supervisory effects are conspicuous elements of previous health promotion programmes that have positively affected QOL status $(3,4)$. The sustainability of previous facility-based, expert-supervised programmes and their beneficial effects beyond the study lifespan may, however, be questioned, once the group and supervisory effects are reduced or removed upon trial termination.

During the informal evaluation of the trial, participants (both exercise and sham exercise groups) highlighted the allocation of specific times during the day, the association of physical exercise sessions to daily routines (such as before tea, after brushing teeth, or during work breaks), and the incorporation of friends and family into exercising with them, as some of the strategies which reinforced engagement with the programme. While the self-set reminders and routines associated with performance of the lifestyle physical exercise enhanced compliance to the exercise programme, the social support accrued from asking friends and relatives to join in the exercise improved the pleasure derived from it, their adherence to it, and their intention to continue with it after the trial.

The SCT has been used successfully in other physical activity interventions targeted at teenage 
girls $(22,28)$, but there has been no (published) use of this theory for bone-health physical exercise interventions among premenopausal women. Hence, in comparisons of efficacy with previous nonfacility-based and non-supervised physical exercise interventions for bone health, the use of the SCT might have strengthened the position of the current study. As in the study by Murphy et al. (28), excellent compliance rates, a high level of positive affect towards the lifestyle physical exercise by both exercise and sham exercise groups, and the positive indication to adopt the exercise beyond the study lifespan are some of the strengths of the present investigation. These indicate that, regardless of potential barriers, premenopausal women are capable of managing their own physical exercise regime when they have been facilitated to develop the relevant lifestyle skills, and do not necessarily need to attend supervised or facility-based programmes before they can engage in physical exercises for health promotion.

Current physical inactivity levels among women are high (29), and the persistence of such scenarios implies a 'time bomb' in the health care and economic system of many nations. For instance, an epidemiological surveillance system in the UK (Health Survey for England; HSE) documented that $29 \%$ of women, compared to $39 \%$ of men, over 16 years engaged in the minimum recommended levels of 30 minutes of moderate physical activity on at least 5 days a week $(29,30)$. Considering, however, inflation in self-reported constructs of this nature (31), the true levels of engagement in physical activity are likely to be much less than that reported in the HSE. This notion is corroborated by the findings that only $4 \%$ of women between the ages of 16 and 34 years reached the recommended physical activity level when objective measures of physical activity were employed in the HSE study. It appears that the impetus from the wealth of evidence dating back to Hippocrates over 2000 years ago (32), widening participation (33), and gender equality initiatives (34) to encourage increased physical activity and exercise engagement is yet to be substantiated in premenopausal female participation in recommended physical activity levels. The reasons (e.g. finances, educational attainment, societal factors) for this health disparity are interrelated (e.g. men with high educational attainment will likely have a higher socioeconomic status, and are more likely to have more time to visit gym facilities compared to female counterparts) and do not appear to have given way to many changes over the years $(35,36)$. In the interim, a relatively low-burden, accessible exercise intervention as identified in this study may be promoted among premenopausal women.

In practice, getting people who are most likely to benefit from exercise-induced augmentation of bone health may present a greater challenge to public health prevention of osteoporosis than the clarification of an optimal exercise prescription. The high level of acceptability of the lifestyle physical exercise in this research, as reflected in the compliance and adherence data, as well as the feedback given by participants, is an indication of its likelihood to succeed as a public bone-health promotion strategy. The design of exercise sessions, which were described by participants as easily adaptable, aimed to circumvent common barriers to engagement in lifestyle physical activity among premenopausal women. Hence, the study lends support to the shift in focus away from a medical research model towards translatable interventions with external validity for population-level augmentation of bone health.

\section{Implications for practice}

Apparently, considerable volumes of regular and high-intensity physical exercise are perceived as barriers and have, unfortunately, been unappealing to many premenopausal women [15]. It may be that a favourable shift in patterns of physical exercise participation among premenopausal women could be stimulated by proof of efficacy of other healthrelated benefits of non-rigorous bone loading exercise programmes. Thus, a practical lifestyle approach to physical exercise for optimisation of bone health in premenopausal women may offer a much-needed public health strategy to reduce overall prevalence of osteoporosis (38). The design of such exercise interventions should also motivate the recipients as well as instituting a paradigm shift from laborious physical activities to pleasurable exercises that also offer maximal osteogenic gains. Therefore, it is important (as in the current study) that studies exploring the efficacy of physical exercise interventions consider both clinical and health-related QOL outcomes. 


\section{Limitations}

The conduct of a lifestyle physical exercise study with a longer period of follow-up may give more credence to the findings of the trial. It is important to note that unforeseen life events, for instance, good news, sickness, or crisis in the family that some participants could have experienced, may affect self-reported QOL among participants. The reliance on self-reported adherence to physical exercise (which is inevitable for a non-facilitybased regime) is a recognised limitation in this study. However, it is envisaged that the effect of this limitation may not invalidate the findings, as participants were requested at the initiation of the study and during follow-up telephone conversations/emails to complete the exercise logs in honesty. The use of an ITT analysis in this study lends support to addressing dropouts and missing data, as analyses on only adherent participants are likely to lead to inflated estimates of exercise intervention effects. The more realistic estimates of exercise intervention effects (derived from ITT analyses) simulate conditions similar to what might likely be recommended in practice for lifestyle physical exercises. This may be of particular relevance for policymakers.

\section{Conclusion}

The relationship between osteogenic lifestyle physical exercises and QOL has not been adequately explored in the general population among premenopausal women without an existing diagnosis of osteoporosis. Hence, the current study serves to fill an important gap in the knowledge base. The results of this study show that bone health optimising exercises, when implemented as easily adoptable lifestyle physical activity, may enhance QOL of premenopausal women. The results have important public health implications: a practical lifestyle approach to physical exercise for optimisation of bone health among premenopausal women may offer a much-needed public health strategy to preventing osteoporosis. The boost, or at least sustenance, of health-related QOL may be one of the most important motivators for people to engage in lifestyle physical exercise, thereby improving bone health as well as their perception of own general health status.
Conflict of interest

None declared.

\section{Funding}

This research received no specific grant from any funding agency in the public, commercial, or not-for-profit sectors.

\section{References}

1. Timmins N. NHS goes to the PROMS. BMJ. 2008; 336: 1464-1465.

2. Gillison FB, Skevington SM, Sato A, Standage M, Evangelidou $S$. The effects of exercise interventions on quality of life in clinical and healthy populations; a meta-analysis. Soc Sci Med. 2009; 68: 1700-1710.

3. Hongo M, Itoi E, Sinaki M, Miyakoshi N, Shimada Y, Maekawa S, et al. Effect of low-intensity back exercise on quality of life and back extensor strength in patients with osteoporosis: a randomized controlled trial. Osteoporosis Int. 2007; 18: 13891395.

4. Li WC, Chen YC, Yang RS, Tsauo JY. Effects of exercise programmes on quality of life in osteoporotic and osteopenic postmenopausal women: a systematic review and meta-analysis. Clin Rehabil. 2009; 23: 888-896.

5. Kohrt W, Bloomfield S, Little K, Nelson M, Yingling V. American College of Sports Medicine Position Stand: physical activity and bone health. Med Sci Sports Exerc. 2004; 36(11): 1985-1996.

6. Karlsson MK, Nordquist A, Karlsson C. Sustainability of exercise-induced increases in bone density and skeletal structure. Food Nutr Res. 2008; 52. DOI: $10.3402 /$ fnr.v52i0.1872

7. Babatunde OO. Primary prevention of osteoporosis: time to redefine the wheel? J Osteoporosis Phys Activ. 2014; 2. DOI: 10.4172/2329-9509.1000e107.

8. Schmiege SJ, Aiken LS, Sander JL, Gerend MA. Osteoporosis prevention among young women: Psychosocial models of calcium consumption and weight-bearing exercise. Health Psychol. 2007; 26: 577-587.

9. Sedlak CA, Doheny MO, Jones SL. Osteoporosis prevention in young women. Orthop Nurs. 1998; 17: 53-60.

10. Olson ACF. Perimenopausal women's intended and actual behavioural response to bone health interventions. ProQuest; Doctoral thesis, University of Arizona, 2008.

11. Kulp JL, Rane S, Bachmann G. Impact of preventive osteoporosis education on patient behaviour: immediate and 3-month follow-up. Menopause 2004; 11: 116-119.

12. Boyd S, Rhodes R, Higgins JW, McKay H, Khan K. A blueprint for bone health across the lifespan: engaging novel team members to influence fracture rates. Br J Sports Med. 2011; 45: 463-464.

13. Winzenberg T, Oldenburg B, Frendin S, De Wit L, Riley M, Jones G. The effect on behavior and bone 
mineral density of individualized bone mineral density feedback and educational interventions in premenopausal women: a randomized controlled trial [NCT00273260]. BMC Public Health. 2006; 6: 12.

14. Sternfeld B, Ainsworth BE, Quesenberry CP. Physical activity patterns in a diverse population of women. Prev Med. 1999; 28: 313-323.

15. Clark J. Women too busy to exercise. BMJ 2003; 326 : 467.

16. Chapurlat R, Garnero P, Sornay-Rendu E, Arlot M, Claustrat B, Delmas P. Longitudinal study of bone loss in pre-and perimenopausal women: evidence for bone loss in perimenopausal women. Osteoporosis Int. 2000; 11: 493-498.

17. Mazess R, Barden H, Ettinger M, Johnston C, Dawson-Hughes B, Baran D, et al. Spine and femur density using dual-photon absorptiometry in US white women. Bone Miner. 1987; 2: 211-219.

18. Pocock N, Eberl S, Eisman J, Yeates M, Sambrook P, Freund J, et al. Dual-photon bone densitometry in normal Australian women: a cross-sectional study. Med J Aust. 1987; 146: 293-297.

19. Rodin A, Murby B, Smith M, Caleffi M, Fentiman I, Chapman M, et al. Premenopausal bone loss in the lumbar spine and neck of femur: a study of 225 Caucasian women. Bone. 1990; 11: 1-5.

20. Babatunde O, Forsyth J. Effects of lifestyle exercise on premenopausal bone health: a randomised controlled trial. J Bone Miner Metab. 2014; 35: 563572. DOI: 10.1007/s00774-013-0527-9.

21. Baranowski T, Perry CL, Parcel GS. How individuals, environments, and health behavior interact. In: Glanz K, Rimer BK and Lewis FM (eds) Health behavior and health education: Theory, research, and practice. $3 \mathrm{rd}$ ed. San Francisco: Jossey-Bass, 2002, pp, 165-184.

22. Dishman RK, Motl RW, Saunders R, Felton G, Ward DS, Dowda M, et al. Self-efficacy partially mediates the effect of a school-based physical-activity intervention among adolescent girls. Prev Med. 2004; 38: 628-636.

23. Babatunde O, Forsyth J, Gidlow C. A meta-analysis of brief high-impact exercises for enhancing bone health in premenopausal women. Osteoporosis Int. 2012; 23: 109-119.

24. Friedlander AL, Genant HK, Sadowsky S, Byl NN, Glüer CC. A two-year program of aerobics and weight training enhances bone mineral density of young women. J Bone Miner Res. 1995; 10: 574-585.

25. Maruish ME, Turner-Bowker DM. A guide to the development of certified modes of short form survey administration. Lincoln, RI: QualityMetric Inc., 2009.

26. Ware JE Jr, Sherbourne CD. The MOS 36-item shortform health survey (SF-36): I. Conceptual framework and item selection. Med Care. 1992; 30: 473-483.
27. Hardman AE, Stensel DJ. Physical activity and health: the evidence explained. London: Taylor \& Francis, 2009.

28. Murphy NM, Ni Dhuinn M, Browne PA, ÓRathaille MM. Physical activity for bone health in inactive teenage girls: is a supervised, teacher-led program or self-led program best? J Adolesc Health. 2006; 39: 508-514.

29. Centers for Disease Control and Prevention. Unpublished estimates from the 2008 National Health Interview Survey. Atlanta, Georgia, United States: Centers for Disease Control and Prevention, 2008.

30. Health and Social Care Information Centre. Health Survey for England 2008 - Physical activity and fitness: summary of key findings. A survey carried out on behalf of the NHS Information Centre, 2008. Available from: https://catalogue.ic.nhs.uk/ publications/public-health/surveys/heal-surv-physacti-fitn-eng-2008/heal-surv-phys-acti-fitn-eng2008-rep-v1.pdf. (Accessed August 15, 2012)

31. Van de Mortel TF. Faking it: social desirability response bias in self-report research. Aust J Adv Nurs. 2008; 25: 40-48.

32. Eaton SB, Shostak M, Konner M. The Paleolithic prescription: a program of diet $\&$ exercise and a design for living. Harper \& Row, 1988.

33. O'Brien L, Morris J. Active England Woodland Projects. The Research Agency for the Forestry Commission: Social and Economic Research Group; 2009. Available from http://www.forestry.gov.uk/ pdf/active_england_final_report.pdf/\$file/active_ england_final_report.pdf. (Accessed March 26, 2012)

34. Sport England. Driving up participation: The challenge for sport: academic review papers commissioned by Sport England as contextual analysis to inform the preparation of the framework for sport in England. Sport England, 2004.

35. Borodulin K, Laatikainen T, Juolevi A, Jousilahti P. Thirty-year trends of physical activity in relation to age, calendar time and birth cohort in Finnish adults. Eur J Public Health. 2008; 18: 339-344.

36. Talbot LA, Fleg JL, Metter EJ. Secular trends in leisure-time physical activity in men and women across four decades. Prev Med. 2003; 37: 52-60.

37. National Institutes of Health. Osteoporosis prevention, diagnosis, and therapy. NIH Consensus Development Panel on Osteoporosis Prevention, Diagnosis, and Therapy. JAMA. 2001; 285: 785795.

38. World Health Organization. Global health risks: mortality and burden of disease attributable to selected major risks. Geneva: Stylus Pub LLC. 